\title{
An Investigation of Ensemble Approaches to Cross-Version Defect Prediction
}

\author{
$1^{\text {st }}$ Xiaoxing Yang, $2^{\text {nd }}$ Xin Li, $3^{\text {rd }}$ Wushao Wen \\ School of Data and Computer Science \\ Sun Yat-Sen University \\ Guangzhou, China \\ (yangxx27@mail,lixin49@mail2,wenwsh@mail).sysu.edu.cn
}

\author{
$4^{\text {th }}$ Jianmin $\mathrm{Su}$ \\ School of Physics and Optoelectronic Engineering \\ Guangdong University of Technology \\ Guangzhou, China \\ jackysura@163.com
}

\begin{abstract}
Software defect prediction can help software testers to focus on software modules with more defects. Many ensemble methods have been proposed for software defect prediction to divide software modules into defect-prone and defect-free, and these ensemble methods have been proved to be more effective than single learning algorithms. A few ensemble approaches have been applied to predict the number of defects in software modules, and they also perform well in most cases. The good performance of ensemble approaches implies that ensemble algorithms might not only improve the accuracy of software defect classification models, but also improve the performance of defect ranking models. Therefore, we propose an ensemble method based on Yang et al.'s learning-to-rank approach in this paper. Experimental results show that the learning-to-rank-based ensemble approach performs better than the single learningto-rank approach, which means that the idea of ensemble can improve the performance of the learning-to-rank approach to sort modules in order of defect count. We also conduct a comparison study of ensemble approaches for cross-version defect prediction over 30 sets of cross-version data, which indicates that the ensemble technique of random subspace is more appropriate than boosting over these experimental data sets.

Index Terms-Software defect prediction; Ensemble approaches; Ranking task; Learning-to-rank
\end{abstract}

\section{INTRODUCTION}

Software testing activities play a key role in software development, which consume a great amount of resources including time, money and personnel [1]. Timely detecting and repairing defects before releasing the products are critical for software quality assurance [2], [3]. Software defect prediction (SDP) employs software metrics (also referred to as features or attributes) to predict the defect information of software modules (classes, files, etc) in order to support software testing activities [4]. The most frequently investigated tasks of SDP include the classification task [3] and the ranking task [4]. We focus on the ranking task in this paper.

SDP for the ranking task, by sorting software modules in order of defect count, can help testers to focus on software modules with more defects and identify defects more quickly [1], [5]-[7]. Firstly, data is collected from software modules according to software metrics, such as 'response for a class' [8]. Subsequently, modelling approaches are used to construct

Corresponding author: Xiaoxing Yang (yangxx27@mail.sysu.edu.cn). DOI reference number: 10.18293/SEKE2019-113 a model based on data from modules with known defect numbers. Finally, the model is used to predict defects of software modules with unknown defect numbers, and thus an order of these modules based on the predicted values is obtained, which can help allocate testing resources (for instance, more testing resources for modules with more defects) [7].

Many methods have been employed to construct SDP models for the ranking task, such as linear regression [9], negative binomial regression [1], recursive partitioning, Bayesian additive regression trees, and random forest [10]. Yang et al. proposed an effective learning-to-rank (LTR) method for this task [4], which constructs SDP models by directly optimizing the ranking performance. That random forest [4], [10] performed well for this task implies the usefulness of ensemble algorithms to improve the performance of defect ranking models. Therefore, in this paper, we investigate an ensemble method based on Yang et al.'s LTR approach, in order to see whether the idea of ensemble can benefit this approach.

Considering that the use of ensemble methods for SDP with ranking task was explored by very few researchers, Rathore and Kumar presented a study of different ensemble methods for the prediction of number of defects over three Eclipse data sets [11]. To further compare the ensemble approaches for this task, we conduct a comparison study of ensemble approaches over 30 sets of cross-version data [7].

The main contributions of this paper include: (a) a novel ensemble method based on Yang et al.'s LTR approach, and a comparison study of the proposed ensemble approach with the single LTR approach [4]; and (b) a comparison study of ensemble approaches for cross-version defect prediction for the ranking task.

The rest of this paper is organized as follows. Section 2 presents related work. In Section 3, we describe the proposed ensemble method. The experimental methodologies are detailed in Section 4 and experimental results are reported in Section 5. Section 6 presents the threats to validity, and Section 7 draws the conclusions.

\section{RELATED WORK}

Data and model construction methods are key factors for software defect prediction(SDP). Because we focus on en- 
semble approaches for cross-version defect prediction for the ranking task, this section mainly presents the related work from two aspects: methods for constructing SDP models for the ranking task, and ensemble methods in SDP.

\section{A. Methods for Constructing SDP Models for the Ranking Task}

Compared with methods for constructing defect prediction models for the classification task, there are fewer approaches particularly proposed for constructing defect prediction models for the ranking task. Most studies employed existing methods to construct models to predict the number of defects. For example, Yang and Wen [7] used two penalized regression methods to construct prediction methods, and experimental results showed that both penalized regression performed better than linear regression and negative binomial regression for cross-version defect prediction. Gao et al. [12] compared eight count models, and the comparative study showed that zeroinflated negative binomial regression, hurdle negative binomial regression and hurdle Poisson regression with threshold 2 were more effective. Weyuker et al. [10] compared four approaches (negative binomial regression, random forest, recursive partitioning and Bayesian additive regression trees) for predicting the number of defects in software modules, and the study showed better performance of negative binomial regression and random forest models than the other two models. Considering that existing SDP models, which are optimized to predict explicitly the number of defects in a software module, might fail to give an accurate order because of the difficulty to predict the exact number of defects in a software module due to noisy data, Yang et al. [4] proposed a LTR approach to directly optimize the ranking performance measure, and the experimental results showed that the LTR approach and random forest were better than other methods to construct SDP models for the ranking task.

\section{B. Ensemble Methods in SDP}

Most existing work of ensemble methods for SDP dealt with classification problems instead of ranking problems. For example, Wang and Yao [13] investigated different types of class imbalance learning methods, including re-sampling techniques, threshold moving, and ensemble algorithms, and they found that AdaBoost.NC showed the best overall performance in terms of the measures including balance, G-mean, and area under the curve. The authors also proposed a dynamic version of AdaBoost.NC to adjust parameter automatically during training. Kumar et al. [14] applied ensemble methods to develop models to predict whether software modules are defect-prone or not using 45 datasets from the PROMISE repository. They concluded that ensemble method learning algorithm outperformed individual classifiers. Yohannese et al. [15] evaluated the capability of ensemble learning algorithms in SDP for the classification task using eight NASA software defect data sets. The experimental results revealed that the combined technique could improve the performance. There are other studies of ensemble methods for SDP for the classification task [16]-[18].

Compared with the work of ensemble methods for defect prediction for the classification task, there exist fewer studies of ensemble methods for defect prediction for the ranking task. A commonly used ensemble method in SDP for the ranking task is random forest. Random forest has been proved to be effective in many studies [4], [10]. Yu et al. [19] explored the potential of using re-sampling techniques and ensemble learning techniques to learn from imbalanced defect data for predicting the number of defects. They studied the use of an ensemble learning technique (i.e., the AdaBoost.R2 algorithm) to handle imbalanced defect data for predicting the number of defects. Experimental results showed the effectiveness of these approaches. Rathore and Kumar [11] performed an empirical study of different homogeneous ensemble methods for predicting the number of faults based on three Eclipse datasets. Experimental results showed that overall ensemble methods produced better performance than a single fault prediction technique. According to their conclusion, random subspace produced better results than other ensemble methods such as boosting and bagging, and decision tree regression was a better base learning technique than multilayer perceptron and linear regression. These studies imply that ensemble algorithms might not only improve the accuracy of software defect classification models, but also improve the performance of defect prediction models for the ranking task. Therefore, we want to investigate the ensemble method that uses the LTR approach as the base learning technique, to investigate whether the idea of ensemble can improve the LTR approach for SDP.

\section{The LEARNING-TO-RANK-BASEd ENSEMBLE APPROACH}

In this section, we briefly describe Yang et al.'s learningto-rank (LTR) approach [4]. Subsequently, we present our proposed ensemble method based on this approach.

\section{A. Yang et al.'s Learning-to-Rank Approach}

Given a vector of metrics of a software module $\mathbf{x}=\left(x_{1}, x_{2}, \cdots \cdots, x_{d}\right),\left(x_{i}\right.$ : the $i^{\text {th }}$ metric, and d: number of metrics), the goal of SDP for the ranking task is to predict its relative defect number, which is denoted as $f(\mathbf{x})$ as Equ.(1).

$$
f(\mathbf{x})=\sum_{i=1}^{d} \alpha_{i} x_{i}
$$

where $\alpha_{i}$ s are parameters obtained by training. Once $\alpha_{i}$ s are fixed, the model is learned. The LTR approach optimizes performance of defect prediction models directly to obtain the parameters using composite differential evolution (CoDE) [20]. Details can be found in Yang et al.'s study [4].

\section{B. The Learning-to-Rank-Based Ensemble Approach}

When adopting the LTR model as the base learner, the values of $f(\mathbf{x})$ can vary greatly in the same ranking performance. For this reason, when computing the final output combining all base learning models, we use sigmoid function 
to transform the predicted values by the base learning models. When the values of $f_{k}(\mathbf{x})$ (the predicted values achieved by the $k^{t h}$ base learning models) are large, the values of the corresponding sigmoid function are similar. In order to better distinguish these values, we set a coefficient $C 1$ for $f_{k}(\mathbf{x})$, which will not affect the monotonicity. In addition, we set different weights for different base learning models according to their performance over the training set. Assuming that $P_{k}$ is the training performance of model $f_{k}(\mathbf{x})$, the final output $G 1(\mathbf{x})$ that combines all base learning models is computed as Equ.(2):

$$
\begin{aligned}
G 1(\mathbf{x}) & =\sum_{k=1}^{N 1} P_{k}^{W} * S\left(f_{k}(C 1 * \mathbf{x})\right) \\
& =\sum_{k=1}^{N 1} P_{k}^{W} /\left(1+e x p^{-C 1 * f_{k}(\mathbf{x})}\right)
\end{aligned}
$$

where $N 1$ is the number of base learning models, and $W$ is the power that controls the contributions of different base learning models to the final output according to their training performance. When $W$ equals to 0 , the contributions of all base learning models to the final output are the same, regardless of the training performance of these base learning models.

Rathore and Kumar's study [11] showed that random subspace produced better results than other ensemble methods such as boosting and bagging, and random forest [21] (which adopts random subspace) has been proved to be effective in many studies [4], [10]. Therefore, we learn from the selection process of random forest. Assuming that there are $d$ metrics, a subset of $N 2$ metrics are selected randomly. Subsequently, the best $N 3$ metrics are selected from the $N 2$ metrics for constructing base learning models. In this paper, we adopt fault-percentile-average (FPA) [10] as the model performance measure, which is detailed in Section IV. The effectiveness of one metric can be evaluated by directly computing the FPA value of the model based on the metric.

The detailed process of the learning-to-rank-based ensemble approach (LTRE) are shown as follows.

1) Input:

M training vectors: $\mathbf{x}_{i}=\left(x_{i 1}, x_{i 2}, \ldots, x_{i d}\right)$, i: 1 to $\mathrm{M}$, $\mathrm{d}$ : number of metrics; Number of base learning models: N1; Number of alternative metrics: N2; Number of used metrics: N3; Coefficient: C1; Power: W.

2) Process:

a) for $j=1,2, \ldots d$

i) compute the performance of model based on training data with only the $j^{\text {th }}$ metric, and obtain the corresponding performance $F_{j}$.

end for

b) for $k=1,2, \ldots N 1$

i) select $\mathrm{N} 2$ metrics from all metrics randomly

ii) select the best N3 metrics from the selected N2 metrics according to $F_{j}$ values iii) use the training vectors with the best $\mathrm{N} 3$ metrics to train a model and obtain its performance:

A) model: $f_{k}(\mathbf{x})=\sum_{j=1}^{N 3} \alpha_{k j} x_{j}$, j belongs to the N3 metrics, $\alpha_{k j}$ s are obtained using CoDE.

B) training performance of model $f_{k}(\mathbf{x}): P_{k}$. end for

3) Output: for a testing vector $\mathbf{x}$, compute the predicted value using Equ.(2)

\section{ExPERIMENTAL Methodologies}

In this section, we detail our experimental data sets, the performance measure and implementation.

\section{A. Datasets}

In order to facilitate others to reproduce results, we use 41 data sets from 11 open-source projects in PROMISE repository [7], [22]. The characteristics of these experimental data sets are shown in Table I. The columns of 'faulty modules', 'range of defects', and 'total defects' respectively record the number of modules having defects (with the percentages of faulty modules in the subsequent brackets), the ranges of defect numbers in the corresponding data sets, and the total number of defects in all modules of the corresponding data sets. The metric number of these data sets is 20. Details of the specific twenty metrics can be found Jureczko et al. 's work [8].

\section{B. Performance Measure}

As mentioned in Yang et al.'s study [4], models with higher prediction accuracy (smaller average absolute errors or relative errors) might give a worse ranking, while fault-percentileaverage (FPA) [10] takes into account both practical use and the whole ranking performance of prediction models, which is consistent with cumulative lift chart [23] for measuring a ranking [4]. Therefore, we adopt FPA as the model performance measure.

Considering $n$ modules $f_{1}, f_{2}, \ldots, f_{n}$, listed in increasing order of predicted defect number, $k_{i}$ as the actual defect number in the module $f_{i}$, and $k=k_{1}+k_{2}+\ldots+k_{n}$ as the total number of defects in all modules, the proportion of actual defects in the top $m$ predicted modules to the whole defects is $\frac{1}{k} \sum_{i=n-m+1}^{n} k_{i}$. Then FPA is defined as follows [10]:

$$
\frac{1}{n} \sum_{m=1}^{n} \frac{1}{k} \sum_{i=n-m+1}^{n} k_{i}
$$

Larger FPA means better ranking performance.

\section{Implementation}

There are two main objectives in this paper:

1) investigating whether the idea of ensemble can improve Yang et al.'s LTR approach;

2) and comparing ensemble approaches for cross-version defect prediction for the ranking task.

According to the two objectives, we first compare the learningto-rank-based ensemble method (LTRE) with the single LTR 
TABLE I

EXPERIMENTAL DATA SETS

\begin{tabular}{lllll}
\hline $\begin{array}{l}\text { Datasets } \\
\text { name }\end{array}$ & $\begin{array}{l}\text { module } \\
\text { number }\end{array}$ & faulty modules & $\begin{array}{l}\text { range of } \\
\text { defects }\end{array}$ & $\begin{array}{l}\text { total } \\
\text { defects }\end{array}$ \\
\hline ant-1.3 & 125 & $20(16 \%)$ & {$[0,3]$} & 33 \\
ant-1.4 & 178 & $40(22.5 \%)$ & {$[0,3]$} & 47 \\
ant-1.5 & 293 & $32(10.9 \%)$ & {$[0,2]$} & 35 \\
ant-1.6 & 351 & $92(26.2 \%)$ & {$[0,10]$} & 184 \\
ant-1.7 & 745 & $166(22.3 \%)$ & {$[0,10]$} & 338 \\
lucene-2.0 & 195 & $91(46.7 \%)$ & {$[0,22]$} & 268 \\
lucene-2.2 & 247 & $144(58.3 \%)$ & {$[0,47]$} & 414 \\
lucene-2.4 & 340 & $203(59.7 \%)$ & {$[0,30]$} & 632 \\
xalan-2.4 & 723 & $110(15.2 \%)$ & {$[0,7]$} & 156 \\
xalan-2.5 & 803 & $387(48.2 \%)$ & {$[0,9]$} & 531 \\
xalan-2.6 & 885 & $411(46.4 \%)$ & {$[0,9]$} & 625 \\
xalan-2.7 & 909 & $898(98.8 \%)$ & {$[0,8]$} & 1213 \\
xerces-init & 162 & $77(47.5 \%)$ & {$[0,11]$} & 167 \\
xerces-1.2 & 440 & $71(16.2 \%)$ & {$[0,4]$} & 115 \\
xerces-1.3 & 453 & $69(15.2 \%)$ & {$[0,30]$} & 193 \\
xerces-1.4 & 588 & $437(74.3 \%)$ & {$[0,62]$} & 1596 \\
camel-1.0 & 339 & $13(3.8 \%)$ & {$[0,2]$} & 14 \\
camel-1.2 & 608 & $216(35.5 \%)$ & {$[0,28]$} & 522 \\
camel-1.4 & 872 & $145(16.6 \%)$ & {$[0,17]$} & 335 \\
camel-1.6 & 965 & $188(19.5 \%)$ & {$[0,28]$} & 500 \\
ivy-1.1 & 111 & $63(56.8 \%)$ & {$[0,36]$} & 233 \\
ivy-1.4 & 241 & $16(6.6 \%)$ & {$[0,3]$} & 18 \\
ivy-2.0 & 352 & $40(11.4 \%)$ & {$[0,3]$} & 56 \\
synapse-1.0 & 157 & $16(10.2 \%)$ & {$[0,4]$} & 21 \\
synapse-1.1 & 222 & $60(27.0 \%)$ & {$[0,7]$} & 99 \\
synapse-1.2 & 256 & $86(33.6 \%)$ & {$[0,9]$} & 145 \\
velocity-1.4 & 196 & $147(75.0 \%)$ & {$[0,7]$} & 210 \\
velocity-1.5 & 214 & $142(66.4 \%)$ & {$[0,10]$} & 331 \\
velocity-1.6 & 229 & $78(34.1 \%)$ & {$[0,12]$} & 190 \\
jedit-3.2 & 272 & $90(33.1 \%)$ & {$[0,45]$} & 382 \\
jedit-4.0 & 306 & $74(24.2 \%)$ & {$[0,23]$} & 226 \\
jedit-4.1 & 312 & $79(25.3 \%)$ & {$[0,17]$} & 217 \\
jedit-4.2 & 367 & $48(13.1 \%)$ & {$[0,10]$} & 106 \\
jedit-4.3 & 492 & $11(2.2 \%)$ & {$[0,2]$} & 12 \\
log4j-1.0 & 135 & $34(25.2 \%)$ & {$[0,9]$} & 61 \\
log4j-1.1 & 109 & $37(33.9 \%)$ & {$[0,9]$} & 86 \\
log4j-1.2 & 205 & $189(92.2 \%)$ & {$[0,10]$} & 498 \\
poi-1.5 & 237 & $141(59.5 \%)$ & {$[0,20]$} & 342 \\
poi-2.0 & 314 & $37(11.8 \%)$ & {$[0,2]$} & 39 \\
poi-2.5 & 385 & $248(64.4 \%)$ & {$[0,11]$} & 496 \\
poi-3.0 & 442 & $281(63.6 \%)$ & {$[0,19]$} & 500 \\
\hline & & & &
\end{tabular}

approach. Subsequently, we conduct a comparison of six ensemble methods over 30 sets of cross-version data: LTRE, random forest [21], Bagging [24], Extra-Trees [25], gradient boosting [26], and Adaboost [27].

Because we use the same data sets as Yang and Wen's paper [7], we directly use their experimental results of both LTR and random forest in this paper. LTRE is implemented in Java. For LTRE, N1 is set to 100, N2 is set to half of all metrics, $\mathrm{N} 3$ is set to $2, \mathrm{C} 1$ is set to 0.1 , and $\mathrm{W}$ is set to 3. Bagging, Extra-Trees, gradient boosting, and Adaboost are implemented in Python, and their parameters are tuned by GridSearchCV method which provides the grid search that exhaustively generates candidates from a grid of parameter values specified in advance. For each method, only a small subset of parameters which play an important role are tuned, while others are set as their default values. Because the best parameters for different data sets are different, we choose the most frequently occurring ones. Bagging uses decision trees as base estimator, the number of trees is set to 200 , and the number of metrics is set to 0.4 of all metrics. To be noted, the number of metrics for Bagging is not all metrics (the performance of all metrics is worse than 0.4 of all metrics), so the Bagging here is not the traditional Bagging [11]. The number of trees in Extra-Trees, gradient boosting and Adaboost is also set to 200, the number of metrics in ExtraTrees and gradient boosting is set to $1 / 3$ of all metrics, and the learning rate in gradient boosting and Adaboost is set to 0.1 .

In order to simulate the actual situation, we adopt crossversion defect prediction, whose merit has been shown in Shukla et al.'s work [22]. SDP models constructed according to one version are used to predict defects of the next version, which is similar to actual use. All methods run 10 times for the same set of data. We use Wilcoxon rank-sum test [28] (which is called ranksum for short) to test whether 10 results by one method is significantly larger than those by another method using the same training and testing data.

\section{EXPERIMENTAL RESULTS}

In this section, we firstly show the comparison of the learning-to-rank-based ensemble approach (LTRE) and the single learning-to-rank approach (LTR) in order to see whether the idea of ensemble can improve LTR for SDP. Subsequently, the results of all compared ensemble approaches for crossversion defect prediction are presented.

\section{A. Comparison of LTRE with LTR}

In this subsection, we compare LTRE and LTR. Experimental results are shown in Table II. 'Ant-1.3-1.4' in Table II means using ant-1.3 as the training set and ant-1.4 as the testing set, and others are similar. Hence, there are totally 30 sets of data (each set includes one training version and one testing version). The columns of 'LTRE' and 'LTR' respectively record their mean FPA testing results, and the column of 'pvalues' records the p-values of ranksum test between 10 results achieved respectively by LTRE and LTR over the same set of data. We use bold type to mark the significantly larger results at the 0.05 significance level.

In Table II, '22' in the row of 'larger times' means that LTRE achieves larger mean FPA values than LTR over 22 sets of data. '18' in the row of 'significantly larger times' means that LTRE achieves significantly larger mean FPA values than LTR at the 0.05 significance level over 18 out of 30 sets of data. From these results, the idea of ensemble can benefit LTR for SDP for the ranking task.

\section{B. Comparison of Six Ensemble Approaches}

In this subsection, we show the experimental results of six ensemble approaches: LTRE, random forest (RF), Bagging, Extra-Trees (ET), gradient boosting (GB), and Adaboost (Ada). Table III shows the mean calculated over ten testing FPA results in ten runs for these methods. We use bold type to mark the largest results.

The best methods for different sets of data are very different: one method achieves best results over some sets of data, but 
TABLE III

Mean fPA Results of All Methods for Cross-version Defect Prediction

\begin{tabular}{|c|c|c|c|c|c|c|}
\hline Datasets & LTRE & $\mathrm{RF}$ & Bagging & ET & GB & Ada \\
\hline ant-1.3-1.4 & 0.5921 & 0.5941 & 0.6043 & 0.6366 & 0.5734 & 0.5781 \\
\hline ant-1.4-1.5 & 0.7789 & 0.6956 & 0.6779 & 0.6325 & 0.5574 & 0.5809 \\
\hline ant-1.5-1.6 & 0.8247 & 0.7656 & 0.7708 & 0.7632 & 0.7107 & 0.7529 \\
\hline ant-1.6-1.7 & 0.8251 & 0.8226 & 0.8191 & 0.8085 & 0.7876 & 0.8203 \\
\hline lucene-2.0-2.2 & 0.7168 & 0.7132 & 0.6535 & 0.6656 & 0.6182 & 0.6467 \\
\hline lucene-2.2-2.4 & 0.6937 & 0.6560 & 0.7870 & 0.7809 & 0.7877 & 0.7604 \\
\hline xalan-2.4-2.5 & 0.6417 & 0.6226 & 0.7654 & 0.7707 & 0.7618 & 0.7591 \\
\hline xalan-2.5-2.6 & 0.6725 & 0.6835 & 0.7812 & 0.7833 & 0.7332 & 0.7716 \\
\hline xalan-2.6-2.7 & 0.5685 & 0.5703 & 0.7648 & 0.7486 & 0.6741 & 0.7781 \\
\hline xerces-init-1.2 & 0.4944 & 0.7470 & 0.8593 & 0.8632 & 0.8553 & 0.8507 \\
\hline xerces-1.2-1.3 & 0.5373 & 0.7159 & 0.8441 & 0.8358 & 0.8230 & 0.8120 \\
\hline xerces-1.3-1.4 & 0.7622 & 0.7275 & 0.8515 & 0.8470 & 0.8431 & 0.8718 \\
\hline camel-1.0-1.2 & 0.6735 & 0.6568 & 0.7760 & 0.7187 & 0.7371 & 0.6393 \\
\hline camel-1.2-1.4 & 0.7738 & 0.7891 & 0.7717 & 0.7841 & 0.7721 & 0.7781 \\
\hline camel-1.4-1.6 & 0.7461 & 0.7652 & 0.5541 & 0.5599 & 0.5430 & 0.5497 \\
\hline ivy-1.1-1.4 & 0.7716 & 0.7850 & 0.7109 & 0.7118 & 0.7116 & 0.6971 \\
\hline ivy-1.4-2.0 & 0.8244 & 0.7834 & 0.6490 & 0.6378 & 0.6364 & 0.6832 \\
\hline synapse-1.0-1.1 & 0.7187 & 0.7070 & 0.6940 & 0.6825 & 0.6570 & 0.6544 \\
\hline synapse-1.1-1.2 & 0.6874 & 0.7042 & 0.5017 & 0.5164 & 0.5137 & 0.4940 \\
\hline velocity-1.4-1.5 & 0.6008 & 0.6733 & 0.6813 & 0.6683 & 0.6572 & 0.6904 \\
\hline velocity-1.5-1.6 & 0.7233 & 0.7582 & 0.7007 & 0.6513 & 0.6527 & 0.6847 \\
\hline jedit-3.2-4.0 & 0.8453 & 0.8610 & 0.7056 & 0.6963 & 0.6772 & 0.6857 \\
\hline jedit-4.0-4.1 & 0.8190 & 0.8438 & 0.6651 & 0.6463 & 0.6509 & 0.6680 \\
\hline jedit-4.1-4.2 & 0.8727 & 0.8555 & 0.7551 & 0.7611 & 0.7611 & 0.7547 \\
\hline jedit-4.2-4.3 & 0.6529 & 0.7227 & 0.6185 & 0.5907 & 0.6149 & 0.6340 \\
\hline $\log 4 \mathrm{j}-1.0-1.1$ & 0.8140 & 0.7819 & 0.6772 & 0.6404 & 0.6803 & 0.6749 \\
\hline $\log 4 \mathrm{j}-1.1-1.2$ & 0.5594 & 0.5577 & 0.5652 & 0.5592 & 0.5653 & 0.5640 \\
\hline poi-1.5-2.0 & 0.7003 & 0.6968 & 0.7360 & 0.7248 & 0.7306 & 0.7227 \\
\hline poi-2.0-2.5 & 0.5811 & 0.5020 & 0.6985 & 0.6998 & 0.6575 & 0.6726 \\
\hline poi-2.5-3.0 & 0.6854 & 0.6785 & 0.7211 & 0.7133 & 0.6970 & 0.7000 \\
\hline maximum times & 8 & 8 & 4 & 5 & 2 & 3 \\
\hline
\end{tabular}

worst results over other sets of data. For example, LTRE achieves best result over ant-1.4-1.5, but worst result over xerces-init-1.2. As a whole, LTRE and random forest achieve maximum mean FPA values in most sets of data (eight for each method). These results imply that LTRE is comparable with other ensemble methods.

Bagging achieves larger mean results than Extra-Trees, gradient boosting, and Adaboost over more data sets. In addition, Extra-Trees and Adaboost perform better than gradient boosting over more data sets. To be noted, as mentioned in Section IV, the number of metrics for Bagging is set to $40 \%$ of all metrics because the performance of all metrics is worse than partial metrics. And thus the Bagging here is actually a combination of bagging and random subspace. This implies that random subspace might be better than boosting methods for these data sets.

\section{Threats To VALIDITY}

In this paper, we investigate cross-version defect prediction based on 41 data sets from 11 projects in PROMISE repository, which reflects that the obtained results are strongly related to the SDP domain. However, there are some threats that may have an effect on our experimental results.

One threat is that only a few parameters of all experimental approaches are tuned in a small range, and the results might be different with other parameters setting.

In addition, the experimental data sets are only a very small part of all data sets, among which there are many data sets based on industrial software systems [12] that are not publicly available. Other data sets might include different metrics and totally different modules. The conclusions over these data sets might not hold for other data sets.

The compared ensemble methods are only a small part of all ensemble methods. We use decision tree regression as the base learner for the compared ensemble methods in this paper. When other base learners are used, the conclusion might be different.

\section{CONCLUSIONS}

Many studies have been conducted to sort software modules according to the number of defects [5], [10]. Random forest performed best in some previous studies [4], [10]. The good performance of random forest for SDP implies that the idea of ensemble might be useful to improve the performance of defect ranking models. Yang et al. proposed a learningto-rank method (LTR) particularly for SDP [4], and their experimental results showed its effectiveness. Therefore, we propose a novel ensemble method based on LTR, which is called the learning-to-rank-based ensemble method (LTRE), in order to see whether the idea of ensemble can also improve LTR for sorting module in order of defect count.

Experimental results over 30 sets of cross-version data show that the proposed LTRE perform better than LTR, which implies that the idea of ensemble can benefit LTR for SDP.

In addition, we conduct a comparison study of six ensemble approaches for cross-version defect prediction for the ranking 
TABLE II

MEAN FPA Results of LTRE AND LTR For CRoss-VERsion DEFECT PREDICTION

\begin{tabular}{l|lll}
\hline Datasets & LTRE & LTR & pvalues \\
\hline ant-1.3-1.4 & 0.5921 & 0.5917 & 0.6771 \\
ant-1.4-1.5 & $\mathbf{0 . 7 7 8 9}$ & 0.7113 & 0.0002 \\
ant-1.5-1.6 & $\mathbf{0 . 8 2 4 7}$ & 0.7331 & 0.0002 \\
ant-1.6-1.7 & $\mathbf{0 . 8 2 5 1}$ & 0.8176 & 0.0002 \\
lucene-2.0-2.2 & $\mathbf{0 . 7 1 6 8}$ & 0.7125 & 0.0028 \\
lucene-2.2-2.4 & $\mathbf{0 . 6 9 3 7}$ & 0.6845 & 0.0002 \\
xalan-2.4-2.5 & 0.6417 & $\mathbf{0 . 6 4 6 7}$ & 0.0002 \\
xalan-2.5-2.6 & $\mathbf{0 . 6 7 2 5}$ & 0.6645 & 0.0006 \\
xalan-2.6-2.7 & 0.5685 & 0.5692 & 0.1212 \\
xerces-init-1.2 & 0.4944 & $\mathbf{0 . 6 2 6 6}$ & 0.0002 \\
xerces-1.2-1.3 & 0.5373 & $\mathbf{0 . 6 7 6 3}$ & 0.0002 \\
xerces-1.3-1.4 & $\mathbf{0 . 7 6 2 2}$ & 0.7190 & 0.0002 \\
camel-1.0-1.2 & $\mathbf{0 . 6 7 3 5}$ & 0.6145 & 0.0002 \\
camel-1.2-1.4 & $\mathbf{0 . 7 7 3 8}$ & 0.7541 & 0.0002 \\
camel-1.4-1.6 & 0.7461 & 0.7451 & 0.2263 \\
ivy-1.1-1.4 & $\mathbf{0 . 7 7 1 6}$ & 0.7489 & 0.0002 \\
ivy-1.4-2.0 & $\mathbf{0 . 8 2 4 4}$ & 0.7949 & 0.0002 \\
synapse-1.0-1.1 & $\mathbf{0 . 7 1 8 7}$ & 0.6661 & 0.0002 \\
synapse-1.1-1.2 & $\mathbf{0 . 6 8 7 4}$ & 0.6305 & 0.0002 \\
velocity-1.4-1.5 & 0.6008 & $\mathbf{0 . 6 1 5 3}$ & 0.0017 \\
velocity-1.5-1.6 & 0.7233 & $\mathbf{0 . 7 4 9 5}$ & 0.0002 \\
jedit-3.2-4.0 & 0.8453 & 0.8447 & 0.4274 \\
jedit-4.0-4.1 & 0.8190 & $\mathbf{0 . 8 2 4 4}$ & 0.0046 \\
jedit-4.1-4.2 & $\mathbf{0 . 8 7 2 7}$ & 0.8692 & 0.0028 \\
jedit-4.2-4.3 & 0.6529 & $\mathbf{0 . 6 9 0 0}$ & 0.0002 \\
log4j-1.0-1.1 & $\mathbf{0 . 8 1 4 0}$ & 0.7996 & 0.0003 \\
log4j-1.1-1.2 & 0.5594 & 0.5561 & 0.1212 \\
poi-1.5-2.0 & $\mathbf{0 . 7 0 0 3}$ & 0.6860 & 0.0028 \\
poi-2.0-2.5 & $\mathbf{0 . 5 8 1 1}$ & 0.5131 & 0.0002 \\
poi-2.5-3.0 & $\mathbf{0 . 6 8 5 4}$ & 0.6526 & 0.0002 \\
\hline larger times & 22 & 8 & \\
significantly larger times & 18 & 7 & \\
\hline & & &
\end{tabular}

task over 41 publicly available data sets. The comparison results show that LTRE can perform comparably with other ensemble methods. LTRE and random forest achieve maximum mean FPA values in most sets of data. Nevertheless, the compared ensemble methods have their own advantages in sorting modules in order of defect count over the experimental data sets. The best methods for different sets of data are very different. Therefore, in our future work, we are going to investigate how to choose the appropriate method according to the characteristics of data.

\section{ACKNOWLEDGMENT}

This work is supported by National Natural Science Foundation of China (Grants No. 61602534) and Fundamental Research Funds for the Central Universities (No.17lgpy122).

\section{REFERENCES}

[1] T. Ostrand, E. Weyuker, and R. Bell, "Predicting the location and number of faults in large software systems," IEEE Transactions on Software Engineering, vol. 31, no. 4, pp. 340-355, 2005.

[2] B. Boehm and V. Basili, "Software defect reduction top 10 list," IEEE Computer, vol. 34, pp. 135-137, 2001.

[3] Z. Xu, J. Liu, X. Luo, and T. Zhang, "Cross-version defect prediction via hybrid active learning with kernel principal component analysis," in 25th IEEE International Conference on Software Analysis, Evolution, and Reengineering, 2018, pp. 209-220.

[4] X. Yang, K. Tang, and X. Yao, "A learning-to-rank approach to software defect prediction," IEEE Transactions on Reliability, vol. 64, no. 1, pp. 234-246, 2015.
[5] M. D'Ambros, M. Lanza, and R. Robbes, "Evaluating defect prediction approaches: a benchmark and an extensive comparison," Empirical Software Engineering, pp. 1-47, 2011.

[6] J. Nam and S. Kim, "Clami: defect prediction on unlabeled datasets," in 30th IEEE/ACM International Conference on Automated Software Engineering, 2015. IEEE/ACM, 2015, pp. 452-463.

[7] X. Yang and W. Wen, "Ridge and lasso regression models for crossversion defect prediction," IEEE Transactions on Reliability, vol. 67, no. 3, pp. 885-896, 2018.

[8] M. Jureczko and L. Madeyski, "Towards identifying software project clusters with regard to defect prediction," in Proceedings of the 6th International Conference on Predictive Models in Software Engineering, ser. PROMISE '10, 2010, pp. 9:1-9:10.

[9] N. Ohlsson and H. Alberg, "Predicting fault-prone software modules in telephone switches," IEEE Transactions on Software Engineering, vol. 22, no. 12, pp. 886-894, 1996.

[10] E. Weyuker, T. Ostrand, and R. Bell, "Comparing the effectiveness of several modeling methods for fault prediction," Empirical Software Engineering, vol. 15, no. 3, pp. 277-295, 2010.

[11] S. Rathore and S. Kumar, "Ensemble methods for the prediction of number of faults: a study on eclipse project," in 11th International Conference on Industrial and Information Systems, 2016, pp. 540-545.

[12] K. Gao and T. Khoshgoftaar, "A comprehensive empirical study of count models for software defect prediction," IEEE Transactions on Reliability, vol. 56, no. 2, pp. 223-236, 2007.

[13] S. Wang and X. Yao, "Using class imbalance learning for software defect prediction," IEEE Transactions on Reliability, vol. 37, no. 3, pp. 356$370,2011$.

[14] L. Kumar, S. Rath, and A. Sureka, "An empirical analysis on effective fault prediction model developed using ensemble methods," in 41th Annual Computer Software and Applications Conference, 2017, pp. 244249.

[15] C. Yohannese, T. Li, M. Simfukwe, and F. Khurshid, "Ensembles based combined learning for improved software fault prediction: a comparative study," in 12th International Conference on Intelligent Systems and Knowledge Engineering, 2017, pp. 1-6.

[16] S. Huda, K. Liu, M. Abdelrazek, A. Ibrahim, S. Alyahya, H. AI-Dossari, and S. Ahmad, "An ensemble oversampling model for class imbalance problem in software defect prediction," accepted by IEEE Access, 2018.

[17] Z. Li, X. Jing, X. Zhu, and H. Zhang, "Heterogeneous defect prediction through multiple kernel learning and ensemble learning," in 2017 International Conference on Software Maintenance and Evolution, 2017, pp. 91-102.

[18] Z. Sun, Q. Song, and X. Zhu, "Using coding-based ensemble learning to improve software defect prediction," IEEE Transactions on Systems, Man and Cybernetics-Part C: Applications and Reviews, vol. 42, no. 6 , pp. 1806-1817, 2012.

[19] X. Yu, J. Liu, Z. Yang, X. Jia, Q. Ling, and S. Ye, "Learning from imbalanced data for predicting the number of software defects," in 28th International Symposium on Software Reliability Engineering, 2017, pp. 78-89.

[20] Y. Wang, Z. Cai, and Q. Zhang, "Differential evolution with composite trial vector generation strategies and control parameters," IEEE Transactions on Evolutionary Computation, vol. 15, no. 1, pp. 55-66, 2011.

[21] L. Breiman, "Random forests," Machine Learning, vol. 45, no. 1, pp. 5-32, 2001.

[22] S. Shukla, T. Radhakrishnan, and K. Muthukumaran, "Multi-objective cross-version defect prediction," Soft Computing, pp. 1-22, 2016.

[23] Y. Jiang, B. Cukic, and Y. Ma, "Techniques for evaluating fault prediction models," Empirical Software Engineering, vol. 13, no. 5, pp 561-595, 2008.

[24] L. Breiman, "Bagging predictors," Machine Learning, vol. 24, no. 2, pp. 123-140, 1996.

[25] P. Geurts, D. Ernst, and L. Wehenkel, "Extremely randomized trees," Machine Learning, vol. 63, no. 1, pp. 3-42, 2006.

[26] J. Friedman, "Greedy function approximation: a gradient boosting machine," The Annals of Statistics, vol. 29, no. 5, pp. 1189-1232, 2001.

[27] Y. Freund and R. Schapire, "A decision-theoretic generalization of online learning and an application to boosting," Journal of Computer and System Sciences, vol. 55, pp. 119-139, 1997.

[28] M. Fay and M. Proschan, "Wilcoxon-mann-whitney or t-test? on assumptions for hypothesis tests and multiple interpretations of decision rules," Statistics Surveys, vol. 4, pp. 1-39, 2010. 